Chirurg 2015 · 86:1153

DOI 10.1007/s00104-015-0113-x

Online publiziert: 5. November 2015

c) Springer-Verlag Berlin Heidelberg 2015

CrossMark

H. Dralle

Universitätsklinik für Allgemein-, Viszeral- und Gefäßchirurgie, Universitätsklinikum

Halle, Medizinische Fakultät, Halle (Saale), Deutschland

\title{
Kalzium- und \\ Parathormonkontrolle nach totaler Thyreoidektomie
}

$$
\begin{aligned}
& \text { - } \text { PTH } \geq 15 \text { pg/ml (G1), } \\
& \text { - PTH } 10-15 \text { pg/ml (G2) und } \\
& \text { - } \text { PTH }<10 \text { pg/ml (G3). }
\end{aligned}
$$

Selberherr A, Scheuba C, Riss P, Niederle B (2015) Postoperative hypoparathyroidism after thyroidectomy: efficient and costeffective diagnosis and treatment. Surgery 157:349-353

\section{Hintergrund und Fragestellung}

Nach totalen Thyreoidektomien treten Hypokalzämien früh-postoperativ in ca. $10-30 \%$ auf. Wegen der zunehmenden Häufigkeit totaler gegenüber subtotaler Thyreoidektomien ist daher die postoperative Hypokalzämie in den Vordergrund des Komplikationsmanagements gerückt. Unsicherheiten bestehen vor allem bezüglich der Art der medikamentösen Behandlung und der Frage, ob eine Substitutionstherapie nur bei Hypokalzämiesymptomen oder bereits bei biochemischem Nachweis einer Hypokalzämie indiziert bzw. sinnvoll ist. Die vorliegende Studie hatte das Ziel, diejenige Wertekonstellation des Serumkalziums und Parathormons (PTH) zu definieren, die am Morgen des 1. postoperativen Tages das früh-postoperative Hypokalzämierisiko am besten voraussagt.

\section{Material und Methoden}

Bei 237 Patienten einer 3-jährigen Evaluation wurden nach totaler Thyreoidektomie wegen benigner Struma am 1. und 2 . postoperativen Tag sowie 1 und 6 Monate später die Serumkalzium- und PTH-Werte bestimmt und hinsichtlich ihres prädiktiven Wertes anhand der PTH-Werte am 1. postoperativen Tag in drei Gruppen eingeteilt:
Intraoperativ wurden die Nebenschilddrüsen (NSD) dargestellt und bei nicht möglichem Durchblutungserhalt eine Autotransplantation durchgeführt. Alle Patienten mit einem PTH von $<10$ pg/ $\mathrm{ml}$ oder Kalzium <2,00 mmol/l erhielten eine orale Standardsubstitution mit Kalzium und Calcitriol, die unabhängig vom Serumkalziumwert beendet wurde, wenn PTH $>15$ pg/ml lag.

\section{Ergebnisse}

Bei $0,8 \%$ der Patienten (2/237) trat ein postoperativ permanent substitutionspflichtiger Hypoparathyreoidismus auf. Die Sensitivität der Cut-off-Werte des PTH am 1. postoperativen Tag von 10 bzw. $15 \mathrm{pg} / \mathrm{ml}$ war 100 und $83 \%$, die Spezifität 92 und 99\%, die Genauigkeit 93 und 95\%. Die Sensitivität, Spezifität, und Genauigkeit der Cut-off-Werte des Serumkalziums von 1,8 und 2,0 $\mathrm{mmol} / \mathrm{l}$ waren am 1 . postoperativen Tag 100 und $80 \%, 70$ und $86 \%, 80$ und $84 \%$. Bei keinem der Patienten in $\mathrm{G} 1(\mathrm{PTH} \geq 15 \mathrm{pg} / \mathrm{ml} ; n=178)$ traten Hypokalzämiesymptome auf. Eine persistierend substitutionspflichtige Hypokalzämie entwickelte sich lediglich bei 2 der 33 Patienten aus Gruppe $3(\mathrm{PTH}<10$ pg/ml).

\section{Diskussion und Fazit}

Die vorliegende Studie lässt Schlussfolgerungen zu, die sich sehr gut für die klinische Praxis in der Routineversorgung total-thyreoidektomierter Patienten eignen.
Die Serumkalzium- und PTH-Werte am Morgen des 1. postoperativen Tages weisen die beste Prädiktion hinsichtlich der Wahrscheinlichkeit einer substitutionspflichtigen Hypokalzämie (PTH $<15$ pg/ml) auf. Nur bei PTH-Werten $<15 \mathrm{pg} / \mathrm{ml}$ sollte eine Kalzium-/Vitamin-D-Kombination solange erfolgen, bis PTH über $15 \mathrm{pg} / \mathrm{ml}$ ansteigt. Es gibt keine Hinweise in der Literatur, dass eine prophylaktische Kalzium-/Vitamin-D-Substitution bei einem PTH > $15 \mathrm{pg} / \mathrm{ml}$ die Entwicklung einer persistierenden Hypokalzämie begünstigt.

Welchen Einfluss die Art und Häufigkeit der durchgeführten NSD-Autotransplantationen auf die Dynamik der Erholung der NSD-Funktion hatten, wurde in der vorliegenden Arbeit nicht untersucht. Da der Einfluss der Autotransplantation auf die langfristige NSD-Funktion aufgrund vorliegender Literaturergebnisse jedoch wahrscheinlich gering ist, kann der angegebene PTH-Wert von $15 \mathrm{pg} / \mathrm{ml}$ als in der klinischen Praxis gut verwendbarer Cut-off für $(<15 \mathrm{pg} / \mathrm{ml})$ bzw. gegen (> $15 \mathrm{pg} / \mathrm{ml}$ ) eine postoperative Kalzium-/Vitamin-D-Substitution angesehen werden.

\section{Korrespondenzadresse}

Prof. Dr. med. Dr. h. c. mult. H. Dralle FRCS, FACS, FEBS

Universitätsklinik für Allgemein-, Viszeral- und Gefäßchirurgie,

Universitätsklinikum Halle, Medizinische Fakultät Ernst-Grube-Straße 40, 06097 Halle (Saale) henning.dralle@uk-halle.de

Interessenkonflikt. H. Dralle gibt an, dass kein Interessenkonflikt besteht. 